\title{
ORIGINAL ARTICLE \\ The influence of orthotic gait training with an isocentric reciprocating gait orthosis on the walking ability of paraplegic patients: a pilot study
}

\author{
M Samadian', M Arazpour², M Ahmadi Bani², A Pouyan³, M Bahramizadeh² and SW Hutchins ${ }^{4}$
}

Objectives: The aim of this study was to evaluate the influence of walking with an isocentric reciprocating gait orthosis (IRGO) by spinal cord injury (SCl) patients on walking speed, distance walked and energy consumption whilst participating in a 12-week gait re-training program.

Methods: Six people with motor complete SCI (mean age 29 years, weight $63 \mathrm{~kg}$ and height $160 \mathrm{~cm}$ with injury levels ranging from T8 to T12) participated in this study. Gait evaluation was performed at baseline and after 4,8 and 12 weeks. Walking speed and heart rate were measured to calculate the resulting physiological cost index (PCI).

Results: Reductions in energy consumption were observed after 4,8 and 12 weeks compared with baseline but were not significant. However, walking distance increased significantly $(P=0.010, P=0.003$ and $P=0.005$, respectively) and also did so during the 8-12-week period $(P=0.013)$. Walking speed also improved, but not significantly.

Conclusion: Intensive gait training with the IRGO improved walking speed and the distance walked by paraplegics, as well as reducing the PCl of walking, as compared with baseline during the whole 12-week period. This indicates that further improvements in these parameters may be expected when utilizing gait training longer than 8 weeks.

Spinal Cord (2015) 53, 754-757; doi:10.1038/sc.2015.60; published online 21 July 2015

\section{INTRODUCTION}

Reciprocating gait orthoses (RGOs) are one type of orthosis offered for therapeutic ambulation by paraplegic patients. ${ }^{1}$ However, the rejection rate leading to discontinuation of RGO use in spinal cord injury (SCI) patients has been reported as being high. ${ }^{2}$ Published rejection rates have been demonstrated to be between 61 and 90\% for children with myelomeningocele ${ }^{3,4}$ and between 46 and 54\% in adults with SCI. ${ }^{5,6}$

Previous studies have also reported that the high energy consumption experienced and the high loads applied to the upper limb joints have been the major problems encountered when walking with an orthosis by this patient group. ${ }^{7}$ Many orthoses have been developed to solve these problems, and gait training that includes walking and standing activities with an orthosis is potentially one of the important points in the rehabilitation of these patients.

One of the main aims of orthotic gait training for SCI patients is the provision of an orthosis that can be used with a low level of energy consumption. ${ }^{8}$ As previous studies have proposed the positive effects of ambulation with orthoses for SCI patients on reduction of physiological and psychological complications, this patient group should ideally facilitate their activities of daily living using a mechanical orthosis while experiencing a low level of fatigue and energy cost. ${ }^{9}$

The design of the isocentric reciprocating gait orthosis (IRGO) was originally based on the concept of facilitating a reciprocal gait pattern for paraplegic patients, and is therefore commonly prescribed for walking and standing by SCI patients. It consists of two knee ankle foot orthoses connected laterally to a superstructure supporting the trunk, and includes hip joints that can be locked into extension once the patient is standing. ${ }^{1}$ The hip joints restrict hip motion in the frontal plane and permit reciprocal hip motion in the sagittal plane. The positive effect of this type of orthosis has been reported by Harvey et al. ${ }^{10}$ Winchester et al. ${ }^{11}$ and Leung et al..$^{9}$ on paraplegic patients.

Harvey et al. ${ }^{10}$ in a comparison between the walkabout orthosis (WO) and an IRGO reported that the speed of walking was faster and energy consumption was lower when using the IRGO compared with the WO in 10 complete SCI patients after 8 weeks of gait training. Winchester et al. ${ }^{11}$ in a comparison between the RGO and IRGO demonstrated the energy costs of walking at comfortable self-selected speeds is lower with the IRGO than the RGO. A study by Leung et al., which compared two types of orthosis on energy consumption in SCI patients used 8 weeks gait training with the orthoses. Arazpour et al. ${ }^{8}$ in a comparison of a powered gait orthosis, an IRGO and a hip knee ankle foot orthosis in SCI patients, demonstrated that speed of walking, the distance walked and the physiological cost index (PCI) improved with both the powered gait orthosis and the IRGO as compared with the hip knee ankle foot orthosis, but they had only 6 weeks of gait training to accommodate themselves to the orthosis. Massucci et al. ${ }^{12}$ reported that a walking speed of $16 \mathrm{~m} \mathrm{~min}^{-1}$ could be achieved after a 6- to 8-week period of walking training with an

${ }^{1}$ Loghman Hakim Hospital, Shahid Beheshti University of Medical Sciences, Department of Neurosurgery, Tehran, Iran; ${ }^{2}$ University of Social Welfare and Rehabilitation Sciences, Orthotics and Prosthetics Department, Tehran, Iran; ${ }^{3}$ Department of Neurosurgery, Firouzgar Hospital, Iran University of Medical Sciences, Tehran, Islamic Republic of Iran and ${ }^{4}$ University of Salford, IHSCR, Faculty of Health \& Social Care, University of Salford, Salford, UK

Correspondence: Dr M Arazpour, Department of Orthotics and Prosthetics, University of Social Welfare and Rehabilitation Science, Kodakyar Street, Daneshjo Boulevard, Evin, Tehran 1985713834, Iran.

E-mail: M.arazpour@yahoo.com

Received 25 October 2014; revised 11 January 2015; accepted 4 March 2015; published online 21 July 2015 
Table 1 Characteristics of the paraplegic patients who participated in this study

\begin{tabular}{|c|c|c|c|c|c|c|c|c|}
\hline Patient & Gender & Age (years) & Height $(\mathrm{cm})$ & Weight (kg) & Level of injury & ASIA score & Time since injury (months) & Walking aid used \\
\hline 1 & Female & 25 & 160 & 57 & T8 & A & 35 & Walker \\
\hline 2 & Female & 43 & 155 & 64 & T12 & B & 7 & Walker \\
\hline 3 & Male & 28 & 175 & 68 & T12 & $B$ & 11 & Walker \\
\hline 4 & Male & 30 & 178 & 65 & T10 & $B$ & 21 & Walker \\
\hline 5 & Male & 24 & 168 & 58 & T8 & $A$ & 18 & Walker \\
\hline 6 & Male & 25 & 175 & 63 & T8 & $B$ & 14 & Walker \\
\hline
\end{tabular}

Abbreviation: ASIA, American Spinal Injury Association.

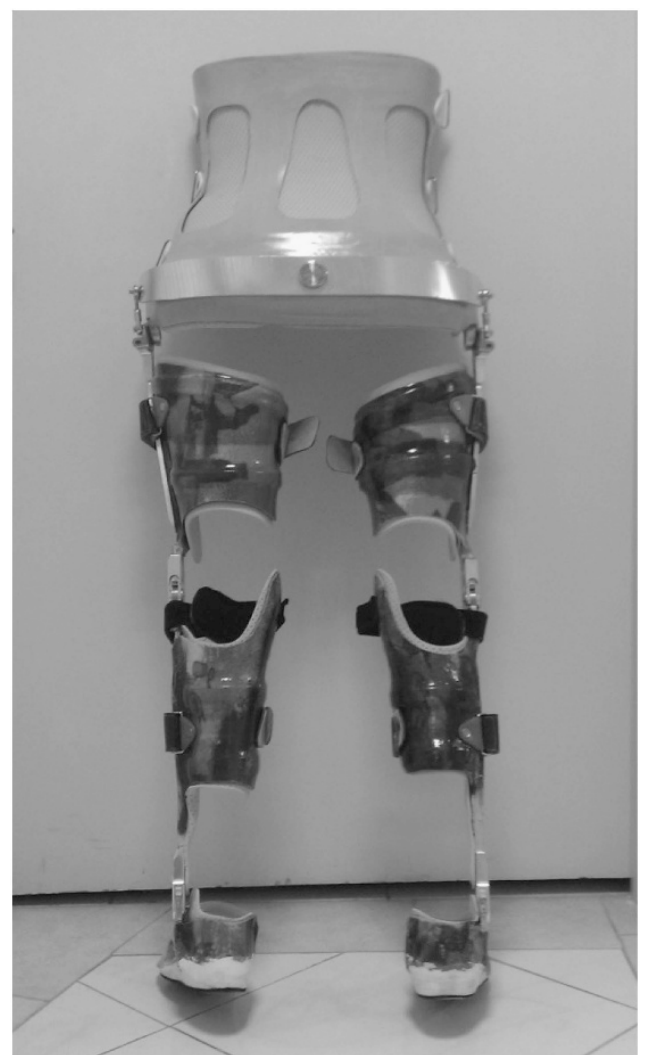

Figure 1 The IRGO used in this study.

advanced reciprocating gait orthosis, but the energy cost of walking increased during this time.

Longitudinal evaluation of the efficiency of orthoses in positively affecting these parameters have not been reported in previous studies in this field, and therefore the objective of the present study was to evaluate the effect of 12 weeks of gait training with an IRGO on speed of walking, distance walked and the PCI demonstrated by paraplegic patients during this extended period. We hypothesized that 12 weeks of concentrated gait training with the IRGO would enable SCI patients to walk more easily with less effort over a longitudinal period and with continued improvement than that previously demonstrated in shorter studies, such as those spanning 8 weeks.

\section{MATERIALS AND METHODS}

\section{Subjects}

Six subjects (four males and two females) with a mean age 29 years, weight $63 \mathrm{~kg}$ and height $160 \mathrm{~cm}$, and with motor complete spinal injury levels ranging from T8 to T12 participate in the study (Table 1). They were characterized by suffering from a traumatic SCI of scale-B or -A according to American Spinal
Injury Association score. They had no previous experience of walking with an orthosis. They also had no evidence of cardiovascular or pulmonary diseases, contractures, severe spasticity, obesity or asymmetric hip positions. Informed consent for the study was obtained from the paraplegic subjects. The ethics committee of University of Social Welfare and Rehabilitation Science approved this study.

\section{Clinical evaluation used in the study}

All evaluations were performed by an orthotist who had been previously trained to administer the evaluations at baseline, after 4, 8 and 12 weeks following the initial supply of the orthosis. The primary outcome measures were walking speed, distance walked and the energy consumption.

Speed of walking was calculated using a stopwatch by measuring the time between starting and stopping time and the distance walked. The volunteer subjects were asked to walk using an IRGO along a pre-determined $40 \mathrm{~m}$ rectangular walkway at their comfortable self-selected speed.

A Polar Heart Rate monitor (Polar Electro, oulu, Finland) was used to gather data to evaluate the PCI. ${ }^{5}$ To calculate the PCI using this method, the heart rate at steady state walking (HRss) and the heart rate at rest (HRar) were recorded, and the following formula was used to measure the PCI (where $V$ is the waking speed): PCI (beats $\left.\mathrm{m}^{-1}\right)=($ HRss - HRar $) / V$.

To obtain a baseline level of PCI, paraplegic patients sat for $10 \mathrm{~min}$ before any measurement. The method of PCI measurement included $5 \mathrm{~min}$ of complete rest in a sitting position, $5 \mathrm{~min}$ in a standing position, walking with the orthosis for $6 \mathrm{~min}$ at their self-selected speed of walking and a final $2 \mathrm{~min}$ of rest in the seated position. During all these activities (that is, during resting, standing and walking), the heart rate was measured every $15 \mathrm{~s}$. After $10 \mathrm{~min}$ of rest in the sitting position, the next evaluation and measurement was started. The mean heart rate during the final $2 \mathrm{~min}$ of rest in the sitting position was considered as HRar and the average heart rate in $6 \mathrm{~min}$ of walking with orthosis was noted as HRss.

\section{Orthotic gait training}

Patients were trained for 12 weeks of gait training after construction of the orthosis that comprised of five sessions per week for a 2-h period with the orthosis. The gait training program also included passive stretching of the lower extremities, upper limb strengthening and balance training with the orthosis while standing and walking. All gait training was applied by a physiotherapist with expertise in walking and standing by paraplegic patients. Approximately $1 \mathrm{~h}$ of the 2-h gait training session was typically taken up by the walking and standing activities. Thirty minutes were needed for the stretching exercises and upper limb strengthening, and a further $30 \mathrm{~min}$ for balance training.

\section{Orthotic intervention}

The IRGO used in this study consisted of two knee ankle foot orthoses that were connected together with a pelvic band. In this orthosis, the bilateral hip joints were connected together via a reciprocal link. The function of the reciprocal link caused flexion of the orthotic hip joint in one side and extension of the hip joint in other side and vice versa. In this study, the ankle foot orthoses incorporated in the IRGO were custom molded using plaster of Paris casts of the lower limbs for each volunteer subject. Figure 1 shows the IRGO used in this study. The volunteer subjects received the IRGO 4 weeks following 
Table 2 Intra-group comparison of speed of walking, walked distance and PCl at baseline and after 4 weeks, 8 weeks and 12 weeks following gait training with IRGO

\begin{tabular}{|c|c|c|c|c|c|c|c|c|c|c|}
\hline & Baseline & After 4 weeks & After 8 weeks & After 12 weeks & $P 1$ & P2 & P3 & P4 & P5 & P6 \\
\hline Walked distance $(\mathrm{m})$ & $86.66 \pm 4.84$ & $88.16 \pm 4.02$ & $90.16 \pm 3.48$ & $93.00 \pm 3.03$ & 0.091 & 0.028 & 0.003 & 0.119 & 0/013 & 0.013 \\
\hline
\end{tabular}

Abbreviation: $\mathrm{PCl}$, physiological cost index.

P1: comparison of variables between baseline and 4th week.

P2. comparison of variables between baseline and 8th week.

P3. comparison of variabs between baseline and 12 week.

P4: comparison of variables between baseline and 12 th week.

P4: comparison of variables between 4th week and 8th week.

P5: comparison of variables between 4 th week and 12th week.

P6: comparison of variables between 8th week and 12th week.

the initial evaluation. Patients were asked to contact the rehabilitation team should they feel any discomfort with the orthosis.

\section{Statistical analysis}

Primary outcome measures were evaluated using a repeated measures analysis of variance. SPSS 16 (SPSS Inc, Chicago, IL, USA) was used for data analysis. The significant level considered was $\alpha=0.05$. Each patient used themselves as a control.

\section{RESULTS}

Comparison of walking speed, distance walked and PCI between 4, 8 and 12 weeks after gait training with an IRGO

A decrease in the PCI of walking when using the IRGO is shown in this study (Table 2). Although not significant, in the intra-group comparison, energy consumption in the paraplegia patients decreased when having received gait training with an IRGO between baseline and 4 weeks, and this continued to decrease up to 12 weeks after IRGO application. However, there were significant improvements in walking speed between baseline and 4 weeks after orthotic gait training $(P=0.010), 8$ weeks after orthotic gait training $(P=0.003)$ and also 12 weeks after walking with the orthosis $(P=0.005)$. There were also significant differences observed in the distance walked after 4, 8 and 12 weeks of orthotic gait training compared with baseline (Table 2). When comparing differences in parameters calculated between 8 and 12 weeks, there was only a statistically significant difference noted for the distance walked (Table 2). Although these results showed a continued improvement during the gait training in energy consumption, this improvement was not statistically significant (Table 2).

\section{DISCUSSION}

The existence of high energy consumption and the problem of experiencing dysfunction in standing and walking due to lack of motor performance, all decrease the ability and capability of performing activities of daily living by paraplegics. The main goal of this study was to assess the effect of gait training on the walking speed and distance walked plus the energy consumption in paraplegic patients over a more extensive period than previously measured. The results indicate a trend rather than an outright improvement in these parameters, which needs more investigation.

In our study, gait training with an IRGO improved the energy consumption experienced compared with baseline. This improvement continued during the whole period of the training time. Their effect probably could be due to the increased endurance and stimulated muscle activity of lower limb muscles with the use of orthosis. Waters et al. ${ }^{13}$ reported there was a positive relationship between the muscle activity of lower extremity and the energy required of the walking with an orthosis. The effect of orthotic gait training with the IRGO on electromyograph of the lower limb muscles was not assessed in this study, and design of a further study will be beneficial in this field.

In addition, a decrease in energy consumption may have occurred for several reasons when performing of orthotic gait training, such as the adoption of an improved trunk position and decreased upper limb joint loading with the orthosis. Reducing energy consumption has been thought to be an important factor for limiting increased symptoms and complications of SCI. In this study the efficiency of orthotic gait training on improving upper limb loading and trunk position was not tested. This needs to be investigated further, as considerable upper limb and trunk movement above the paralysis level is needed to provide walking in SCI patients with a thoracic lesion. ${ }^{14-15}$

In this study the mean improvement in walking speed was 17 $\mathrm{m} \mathrm{min}^{-1}$, which proved to be statistically significant, and continued improvement occurred throughout the training period. This finding indicated that orthotic gait training with an orthosis was capable of improving walking over a period of 12 weeks. Further studies are warranted over a longer training time than 12 weeks to confirm whether any further improvements in gait parameters using an orthosis would be justified given the increasing costs associated with healthcare.

\section{Comparison with other studies}

The results of this study show that energy consumption after 12 weeks of gait training with an IRGO was better than the values reported in previous studies with mechanical orthosis in paraplegic patients. Although many researchers have demonstrated that an extremely high level of exertion is needed when walking with an orthosis in this patient group, ${ }^{9-11}$ it is also clear that SCI patients with high levels of spinal lesion have difficulty in walking in the early stages of orthotic gait training. ${ }^{16}$ However, longitudinal gait training with longer periods have enabled them to walk independently and reciprocally with less level of energy consumption and faster speeds of walking in this study.

The mean speed of walking has been reported to be $0.34 \mathrm{~m} \mathrm{~s}^{-1}$ after 8 weeks of gait training with an orthosis. ${ }^{10}$ In a comparison of different types of RGO in providing adequate walking speed by SCI patients, it has been reported that IRGO provides the highest speed. ${ }^{1}$ Arazpour et al. ${ }^{17}$ reported a gait speed of $0.4 \mathrm{~m} \mathrm{~s}^{-1}$ after 6 weeks of gait training with a powered gait orthosis in four SCI patients.

The literature shows that energy consumption is reported to be less in IRGOs than when wearing hip knee ankle foot orthoses and other RGOs, but an IRGO cannot be a totally suitable replacement for wheelchairs in SCI patients for mobility. ${ }^{1}$ Only $75 \%$ of users of RGOs and HGOs use orthosis for exercise after 6 months of use. ${ }^{12}$ This study has shown that there was only a trend in energy reduction rather than 
a significant one but this occurred as early as 4 weeks after commencing gait training.

\section{Limitations}

There are some limitations to the present study that should be reported. The evaluation of orthotics gait training on patients' quality of life and activity of daily living and also alteration to the subjects' spastic muscle tone were not analyzed in this study, and are recommended for a more longer-term study following this pilot study. Comparison between gait training with IRGO and another assistive device (for example, hip knee ankle foot orthoses, medial linkage orthoses and HGOs) will be beneficial in a further study in this field.

\section{CONCLUSION}

This study demonstrated that during a longitudinal period of orthotic gait training with an IRGO, patients with SCI improved their walking speed, the distance walked and also reduced their energy consumption. In conclusion, patients with SCI walked faster and more efficiently following and during gait training with an IRGO. Therefore, the use of specific gait training programs in the rehabilitation of walking in SCI patients should be considered in conjunction with specific orthoses.

\section{DATA ARCHIVING}

There were no data to deposit.

\section{CONFLICT OF INTEREST}

The authors declare no conflict of interest.

\section{ACKNOWLEDGEMENTS}

We thank the University of Social Welfare and Rehabilitation Sciences for financial support for this research.
1 Arazpour M, Bani MA, Hutchins SW. Reciprocal gait orthoses and powered gait orthoses for walking by spinal cord injury patients. Prosthet Orthot Int 2013; 37: 14-21.

2 Johnson W, Fatone S, Gard S. Walking mechanics of persons who use reciprocating gait orthoses. J Rehabil Res Dev 2009; 46: 435.

3 Katz-Leurer M, Weber C, Smerling-Kerem J, Rottem H, Meyer S. Prescribing the reciprocal gait orthosis for myelomeningocele children: a different approach and clinical outcome. Pediatr Rehabil 2004; 7: 105-109.

4 Sykes L, Edwards J, Powell ES, Ross ER. The reciprocating gait orthosis: long-term usage patterns. Arch Phys Med Rehabil 1995; 76: 779-783.

5 Franceschini M, Baratta S, Zampolini M, Loria D, Lotta S. Reciprocating gait orthoses: a multicenter study of their use by spinal cord injured patients. Arch Phys Med Rehabil 1997; 78: 582-586

6 Jaspers P, Peeraer L, Van Petegem W, Van Der Perre G. The use of an advanced reciprocating gait orthosis by paraplegic individuals: a follow-up study. Spinal Cord 1997; 35: 585-589.

7 Arazpour M, Chitsazan A, Hutchins SW, Ghomshe FT, Mousavi ME, Takamjani EE et al. Design and simulation of a new powered gait orthosis for paraplegic patients. Prosthet Orthot Int 2012; 36: 125-130.

8 Arazpour M, Bani M, Hutchins S, Jones R. The physiological cost index of walking with mechanical and powered gait orthosis in patients with spinal cord injury. Spinal Cord 2012; 51: 356-359.

9 Leung A, Wong A, Wong E, Hutchins S. The physiological cost index of walking with an isocentric reciprocating gait orthosis among patients with $\mathrm{T} 12-\mathrm{L} 1$ spinal cord injury. Prosthet Orthot Int 2009; 33: 61-68.

10 Harvey L, Davis G, Smith M, Engel S. Energy expenditure during gait using the walkabout and isocentric reciprocal gait orthoses in persons with paraplegia. Arch Phys Med Rehabil 1998; 79: 945-949.

11 Winchester P, Carollo J, Parekh R, Lutz L, Aston J. A comparison of paraplegic gait performance using two types of reciprocating gait orthoses. Prosthet Orthot Int 1993; 17: $101-106$.

12 Massucci M, Brunetti G, Piperno R, Betti L, Franceschini M. Walking with the advanced reciprocating gait orthosis (ARGO) in thoracic paraplegic patients: energy expenditure and cardiorespiratory performance. Spinal Cord 1998; 36: 223-227.

13 Waters RL, Adkins R, Yakura J, Vigil D. Prediction of ambulatory performance based on motor scores derived from standards of the American Spinal Injury Association. Arch Phys Med Rehabil 1994; 75: 756.

14 Nene A, Patrick J. Energy cost of paraplegic locomotion using the ParaWalker-electrical stimulation" hybrid" orthosis. Arch Phys Med Rehabil 1990; 71: 116.

15 Hirokawa S, Grimm M. Energy consumption in paraplegic ambulation using the reciprocating gait orthosis and electric stimulation of the thigh muscles. Arch Phys Med Rehabil 1990; 71: 687.

16 Miller N, Merritt J, Merkel K, Westbrook P. Paraplegic energy expenditure during negotiation of architectural barriers. Arch Phys Med Rehabil 1984; 65: 778.

17 Arazpour M, Bani MA, Kashani RV, Ghomshe FT, Mousavi ME, Hutchins SW. Effect of powered gait orthosis on walking in individuals with paraplegia. Prosthet Orthot Int 2013; 37: 261-267. 